Muro de la Investigación, 2021(2), julio-diciembre ISSN: 2523-2886

DOI:https://doi.org/10.17162/rmi.v6i2.1635

\title{
Uso problemático de internet y problemas emocionales y conductuales en estudiantes del nivel secundaria de una institución educativa pública de Lima Norte
}

\section{Problematic Internet use and emotional and behavioral problems in high school students from a public educational institution in the north of Lima}

\author{
Loysi Pezo Cardenas ${ }^{1 \mathrm{a}}$ y Flor Victoria Leiva Colos $^{2}$ \\ Universidad Peruana Unión, Lima, Perú ${ }^{12}$
}

Recibido: 08 de setiembre de 2020

Aceptado: 19 de febrero de 2021

\section{Resumen}

La presente investigación ${ }^{b}$ tuvo como propósito estudiar la relación entre el uso problemático de internet y los problemas emocionales y conductuales en estudiantes del nivel secundario de una Institución Educativa Pública de Lima Norte. La investigación tuvo un diseño no experimental de corte transversal, la muestra estuvo conformada por 539 estudiantes del nivel secundario. Para la recolección de datos se aplicó la escala de uso problemático de internet en adolescentes (EUPI-a), y la escala de Problemas emocionales y conductuales (Youth Self Report) creado originalmente por Achenbach y Rescorla y adaptada a población mexicana por Andrade-Palos, Betancourt-Ocampo y Vallejo-Casarín (2010), posteriormente adaptada y validada a población peruana por BarretoMori (2018) en San Juan de Lurigancho, Lima. Los resultados demostraron que existe una correlación significativa entre uso problemático de internet y problemas emocionales $\mathrm{y}$ conductuales $(\mathrm{rho}=0,200 ; \mathrm{p}=0,000)$. Asimismo, se encontró una correlación significativa entre el uso problemático de internet y las dimensiones de problemas emocionales y conductuales $(p=0,000<.05)$, a excepción de la dimensión de lesiones autoinfligidas $(p=0,344>.05)$.

Palabras clave: Internet, problemas emocionales, problemas conductuales, adolescentes, psicología, autolesión.

\begin{abstract}
The purpose of this research was to study the relationship between the problematic use of the Internet and emotional and behavioral problems in secondary school students from a Public Educational Institution in Lima Norte. The research had a non-experimental cross-sectional design, the sample consisted of 539 high school students. For data collection, the scale of problematic internet use in adolescents (EUPI-a) and the scale of emotional and behavioral problems (Youth

${ }^{a}$ Correspondencia a la autora:

E-mail: loysi.pezo@upeu.edu.pe

bLa presente investigación se basa en la tesis de maestría de las autoras: http://hdl.handle.net/20.500.12840/3153
\end{abstract}


Self Report) originally created by Achenbach and Rescorla and adapted to the Mexican population by Andrade-Palos, Betancourt-Ocampo and Vallejo-Casarín (2010), later adapted and validated to the Peruvian population by Barreto-Mori (2018) in San Juan de Lurigancho, Lima. The results showed that there is a significant correlation between problematic internet use and emotional and behavioral problems (rho $=0.200 ; \mathrm{p}=0.000$ ). Likewise, a significant correlation was found between problematic internet use and the dimensions of emotional and behavioral problems $(\mathrm{p}=$ $0.000<.05)$, with the exception of the dimension of self-inflicted injuries $(p=0.344>.05)$.

Keywords: Internet, emotional problems, behavioral problems, adolescents, psychology, selfharm.

\section{Introducción}

Actualmente, la sociedad es testigo del crecimiento acelerado de la tecnología y mediante sus herramientas podemos adquirir contenidos informativos, transmitir datos, imágenes, música, comunicación local, nacional e internacional; muchas de estas Tecnologías de la Información y la Comunicación (TIC) necesitan el uso de internet; a las que se puede acceder a través de aplicaciones o servicios en el móvil, ordenador o tableta (Sánchez et al., 2015).

En el año 2015, a nivel mundial se registraron 4.700 millones de personas suscriptoras de telefonía móvil, 3.174 millones de habitantes, que equivalía al 43,4\% de la población mundial utilizaban internet y más de 4.200 millones de suscripciones a banda ancha fija y móvil, según la Comisión Económica para América Latina y el Caribe (CEPAL, 2015). Por su parte, Bermúdez (2018) considera que en Sudamérica también existe un nivel de penetración del uso de internet, siendo Argentina considerado el país con mayor porcentaje de uso de internet 93,1\%, seguido de Paraguay 89,6\%, Uruguay 88,2\%, Ecuador 79,9\%, Chile 77,5\%, Brasil 70,7\%, Perú 67,6\%, Bolivia $67,5 \%$, Colombia 63,2\%, Surinam 59,8\%, Venezuela 53,1\%, Guyana 50,5\% y Guyana Francesa $41,4 \%$.

Mientras tanto, en el Perú, la problemática de los adolescentes sobre el uso de internet se visualiza tomando en cuenta el sexo y la edad; puesto que, un 78,2\% de los varones entre 15 y 24 años utilizan más internet que las mujeres de ésta misma edad con un 75,6\%. Es importante destacar que el uso del internet cada día se va incrementando a más temprana edad, los niños, niñas y adolescentes (NNA) menores de 15 años se conectan a internet por temas de entretenimiento, información y comunicación en un 42\%; situación que se vislumbra puesto que el $26 \%$ de ésta población entre 6 a 17 años tienen conexión a internet en sus hogares (El Comercio, 2016); en comparación a datos mencionados por el Instituto Nacional de Estadística e Informática (INEI) 
(2018), mediante la Encuesta Nacional de Hogares (ENAHO) realizado de manera trimestral, en este caso en los meses de enero a marzo (2018) refiere que, el 51,0\% de la población peruana de 6 y más años de edad hizo uso de internet; respecto a similar trimestre de 2017, se observa una disminución de 1,6 puntos porcentuales al pasar de 52,6\% a 51,0\%, mientras que por área de residencia, en Lima Metropolitana el 70,9\% de la población utilizó internet, en el resto urbano el $55,6 \%$ y en el área rural el 13,2\%

La adicción a internet fue cambiada a un nombre de menor debate: Uso Problemático de Internet (UPI) por Shapira et. al (2000) y Chang y Hung (2017) definiéndose como la preocupación constante por el uso de internet, descuidando el tiempo prudente de su uso, así también afirmó que el uso de internet produce un cambio o deterioro del comportamiento. De igual manera, el uso problemático de internet puede definirse como aquel que produce conflictos en cuanto a aspectos psicológicos y sociales los cuales traen consecuencia tanto en entornos escolares, laborales y familiares del individuo (Mellizo, 2017).

Según Cruz (2015) existen excepciones en cuanto al diagnóstico de un uso problemático de Internet, con las personas que hacen uso de éste por motivos de trabajo, porque esto implica un proceso de aprender al máximo en el menor tiempo posible. Araujo (2015) menciona que, para considerar adicción a Internet, la persona debe pasar más de 40 horas a la semana y con sesiones que pueden durar hasta 20 horas seguidas. Por su parte, Luengo (2004) establece los siguientes criterios de diagnóstico: cambios drásticos en los hábitos de vida a fin de tener más tiempo para conectarse, disminución generalizada de la actividad física, descuido de la salud propia a consecuencia de la actividad en Internet, evitación de actividades importantes a fin de disponer de mayor cantidad de tiempo para permanecer conectado.

El uso problemático de internet ha sido asociado a diversas variable, Rial et al. (2014) lo asociaron a diferentes variables sociodemográficas, tales como el papel de los padres, el rendimiento académico, hábitos de uso y consecuencias asociadas; además, Cheung y Wong (2011) señalaron que personas con adicción a internet presentan simultáneamente insomnio y depresión. Del mismo modo, Moral y Fernández (2019) refirieron que el uso problemático de internet en adolescentes se relaciona con la autoestima e impulsividad, indicando que la baja autoestima y la impulsividad cognitiva predicen con mayor probabilidad el uso inapropiado de internet. 
Con los avances tecnológicos, la población con mayor probabilidad de vulnerabilidad son los niños y adolescentes, debido a que muchas veces no son conscientes del uso adecuado de internet y es así que, todo esto va causando un desajuste en su desarrollo integral. Por tanto, Herrera (1999) refiere que la adolescencia es un período crítico debido a los diferentes cambios en el desarrollo físico, psíquico y social como también en el ámbito familiar, es decir, se considera como una etapa de riesgo en el cual puede iniciarse síntomas, enfermedades o alteraciones de la personalidad. Uno de los problemas percibidos hoy en día es el uso de internet que está causando muchos efectos negativos en la sociedad, siendo los adolescentes la población más vulnerable a sufrir graves consecuencias psicosociales y del mismo modo afectando su fortalecimiento emocional, intelectual y personal (García, López \& García, 2014).

Marco y Chóliz (2012) refieren que los adolescentes presentan mayor riesgo a padecer de problemas por el uso inadecuado de internet, por la característica principal de su etapa, la vulnerabilidad, usando tecnología que más allá de su curiosidad por descubrir todas las funciones que esta presenta, también consideran que es el mejor medio para relacionarse con los demás. Por lo expuesto, el presente trabajo de investigación pretende determinar la relación entre el uso problemático de internet y problemas emocionales y conductuales en estudiantes del nivel secundario de una Institución Educativa Pública de Lima Norte.

\section{Metodología}

El presente estudio fue de alcance correlacional, debido a que existió el análisis de la relación entre el uso problemático de internet y los problemas emocionales y conductuales. Asimismo, fue de corte transversal, ya que se recolecto la información en un único momento (Hernández, Fernández \& Baptista, 2014). Su diseño es no experimental, porque no existe la manipulación del investigador en ninguna de las variables, por lo tanto, los datos fueron tomados conforme se encontraron.

La población de estudio estuvo conformada por 507 estudiantes del nivel secundario de ambos sexos, con edades comprendidas entre 11 y 17 años de edad, de nacionalidad peruana pertenecientes al distrito de Los Olivos. Por otro lado, se llevó a cabo en el segundo periodo del año lectivo (2019) en el mes de diciembre, en las instalaciones de una institución educativa pública en el distrito de Los Olivos en la ciudad de Lima. 
Se utilizó La escala de Uso problemático de internet en adolescentes, que tiene como sílabas EUPI-a, fue elaborado por Antonio Rial Boubeta; Patricia Gómez Salgado; Manuel Isorna Folgar; Manuel Araujo Gallego y Jesús Varela Mallou, el presente instrumento mide de manera cualitativa y cuantitativa, está compuesta por 11 ítems con un formato de respuesta tipo Likert de 5 puntos, desde 0 (Nada de acuerdo) a 4 (Totalmente de acuerdo) con un puntaje global comprendido entre 0 y 44 puntos.

También se hizo uso de la escala de Problemas emocionales y conductuales (Youth Self Report) creado originalmente por Achenbach y Rescorla (2001) y adaptada a población mexicana por Andrade-Palos, Betancourt-Ocampo y Vallejo-Casarín (2010), posteriormente adaptada y validada a población peruana por Barreto (2018) en San Juan de Lurigancho-Lima; su objetivo principal es evaluar la sintomatología de 7 problemas tanto emocionales y conductuales, está compuesta por 38 ítems, clasificadas en 7 dimensiones: depresión, rompimiento de reglas, conducta agresiva, consumo de alcohol y tabaco, problemas somáticos y lesiones autoinfligidas.

El tiempo de aplicación del instrumento fue de 10 minutos aproximadamente, con cuatro opciones de respuestas: nunca, pocas veces, muchas veces y siempre, valorados del 1 al 4 respectivamente, se interpreta de la siguiente manera: puntuaciones menores de 50 puntos indican presencia de conductas problema baja, puntuaciones entre 50 a 70 indican moderada presencia de conductas problema y, por último, puntuaciones mayores de 70 indican severa presencia de problemas emocionales y conductuales.

Además, la aplicación puede ser de manera individual y colectiva en población adolescente entre 12 y 18 años. Para ello, se elaboró una ficha de registro de características sociodemográficas donde se solicitaron los siguientes: sexo, edad, lugar de origen, personas con las que conviven, cantidad de ingresos semanales a internet, duración de los ingresos y grado de estudio.

Para la recolección de datos, se procedió a la solicitud de permiso ante las autoridades institucionales, con la finalidad que otorguen la autorización de realizar el trabajo de campo en la institución educativa objeto de estudio, y de esta manera acceder a la recopilación de la información. Se utilizó la Escala de Uso Problemático de Internet en adolescentes (EUPI-a) para evaluar el uso problemático de internet y la Escala de Problemas Emocionales y Conductuales para evaluar la sintomatología de problemas tanto emocionales como conductuales, dichos instrumentos fueron respondidos de manera escrita, previa lectura sin la ayuda de personal externo. 
El procesamiento y análisis de datos se realizó a través del paquete estadístico SPSS versión 25. Los análisis que se realizaron fueron descriptivos e inferenciales, porque se darán a través de tablas para determinar la asociación entre uso problemático de internet y problemas emocionales y conductuales.

\section{Resultados}

\section{Análisis descriptivos de uso problemático de internet}

En la tabla 1 se muestra que el $75 \%$ de los estudiantes presentan un uso problemático de internet, es decir, los adolescentes presentan una conducta placentera que se convierte en una "adicción" ocasionando en la persona una falsa identidad, sustituyendo su mundo exterior por una realidad virtual; por otro lado, solo el $25 \%$ hace un uso no problemático del internet.

\section{Tabla 1}

Uso problemático de internet

\begin{tabular}{lll}
\hline & $\mathbf{n}$ & $\mathbf{\%}$ \\
\hline Uso no problemático & 127 & 25,0 \\
Uso problemático & 380 & 75,0 \\
Total & $\mathbf{5 0 7}$ & $\mathbf{1 0 0 , 0}$ \\
\hline
\end{tabular}

\section{Análisis descriptivo de problemas emocionales y conductuales}

En la tabla 2 se muestra que el 30,8\% de los estudiantes presentan conductas problemáticas severas, es decir, los adolescente presentan problemas internalizados, que son un conjunto de síndromes que se mantienen de manera encubierta y se manifiesta en el interior de la persona; así mismo, problemas externalizados, las que son manifestaciones de carácter externo que afecta las relaciones interpersonales y al mismo tiempo la transgresión de las normas de convivencia social; en cuanto a las conductas problemáticas moderadas se ubican en un 49,5\%; mientras que el 19,7\% presenta conductas problemáticas baja. 
Tabla 2

Problemas emocionales y conductuales

\begin{tabular}{lll}
\hline & $\mathbf{n}$ & $\mathbf{\%}$ \\
\hline Conductas problemáticas baja & 100 & 19,7 \\
Conductas problemáticas moderadas & 251 & 49,5 \\
Conductas problemáticas severa & 156 & 30,8 \\
Total & $\mathbf{5 0 7}$ & $\mathbf{1 0 0 , 0}$ \\
\hline
\end{tabular}

\section{Problemas emocionales y conductuales: depresión}

En la tabla 3 se muestra los problemas emocionales y conductuales en su dimensión depresión por parte de los estudiantes, donde el $34,1 \%$ presenta conductas problemáticas severas, es decir, expresan una visión negativa de su entorno y su futuro; por otro lado, el 36,3\% y el 29,6\% evidencian conductas problemáticas bajas y moderadas, respectivamente.

\section{Tabla 3}

Problemas emocionales y conductuales en la dimensión depresión

\begin{tabular}{lll}
\hline & n & \% \\
\hline Conductas problemáticas baja & 184 & 36,3 \\
Conductas problemáticas moderadas & 150 & 29,6 \\
Conductas problemáticas severa & 173 & 34,1 \\
Total & $\mathbf{5 0 7}$ & $\mathbf{1 0 0 , 0}$ \\
\hline
\end{tabular}

\section{Problemas emocionales y conductuales: rompimiento de reglas}

En la tabla 4 se evidencia los problemas emocionales y conductuales en su dimensión rompimiento de reglas, los cuales un 45,2\% presentan conductas problemáticas severas, es decir, los adolescentes transgreden las normas y estas se manifiestan a través de conductas desadaptativas; mientras que el $43,0 \%$ presentan conductas problemáticas moderadas. 


\section{Tabla 4}

Problemas emocionales y conductuales en la dimensión rompimiento de reglas

\begin{tabular}{lll}
\hline & $\mathbf{n}$ & $\mathbf{\%}$ \\
\hline Conductas problemáticas baja & 60 & 11,8 \\
Conductas problemáticas moderadas & 218 & 43,0 \\
Conductas problemáticas severa & 229 & 45,2 \\
Total & $\mathbf{5 0 7}$ & $\mathbf{1 0 0 , 0}$ \\
\hline
\end{tabular}

\section{Problemas emocionales y conductuales: conducta agresiva}

En la tabla 5 se observa que en cuanto a los problemas emocionales y conductuales en su dimensión conducta agresiva, los adolescentes presentan un $27 \%$ en cuanto a conductas problemáticas severa, mostrando actitudes negativas frente a los demás, como: crueldad y daño a otras personas, asaltos, robo con uso de fuerza, vandalismo, destrozos y daños materiales en casas ajenas, robo de vehículos, huidas de casa y uso extenso de drogas; mientras tanto un 39,6\% evidencia conductas problemáticas moderadas.

\section{Tabla 5}

Problemas emocionales y conductuales en la dimensión conducta agresiva

\begin{tabular}{lll}
\hline Niveles & $\mathrm{n}$ & $\%$ \\
\hline Conductas problemáticas baja & 169 & 33,3 \\
Conductas problemáticas moderadas & 201 & 39,6 \\
Conductas problemáticas severa & 137 & 27,0 \\
Total & 507 & 100,0 \\
\hline
\end{tabular}

\section{Problemas emocionales y conductuales: consumo de alcohol y drogas}

La tabla 6 presenta los problemas emocionales y conductuales en su dimensión consumo de alcohol y tabaco, evidenciando con conductas problemáticas severas a un 13,4\% de estudiantes, es decir, que el consumo de grandes cantidades de sustancias psicoadictivas como el alcohol y tabaco que, aunque puedan ser de poca frecuencia, son significativas por que el consumo se da hasta llegar a la embriaguez en el caso del alcohol; mientras que un 43,4\% muestran conductas problemáticas moderadas. 
Tabla 6

Problemas emocionales y conductuales en la dimensión consumo de alcohol y tabaco

\begin{tabular}{lll}
\hline & $\mathrm{n}$ & $\%$ \\
\hline Conductas problemáticas baja & 219 & 43,2 \\
Conductas problemáticas moderadas & 220 & 43,4 \\
Conductas problemáticas severa & 68 & 13,4 \\
Total & 507 & 100,0 \\
\hline
\end{tabular}

\section{Problemas emocionales y conductuales: problemas somáticos}

En la tabla 7 se muestra los problemas emocionales y conductuales en su dimensión problemas somáticos, demostrando que un 33,5\% presenta conductas problemáticas severas, haciendo uso de mecanismos de defensa que de manera inconsciente puede convertir sus síntomas o malestares emocionales en quejas físicas, alejando su atención de aquellos problemas que le generan ansiedad; mientras que un 36,5\% presenta conductas problemáticas moderadas.

Tabla 7

Problemas emocionales y conductuales en la dimensión problemas somáticos

\begin{tabular}{lll}
\hline & $\mathrm{n}$ & $\%$ \\
\hline Conductas problemáticas baja & 152 & 30,0 \\
Conductas problemáticas moderadas & 185 & 36,5 \\
Conductas problemáticas severa & 170 & 33,5 \\
Total & 507 & 100,0 \\
\hline
\end{tabular}

\section{Problemas emocionales y conductuales: problemas de pensamiento}

En la tabla 8 se muestra los problemas emocionales y conductuales en su dimensión problemas de pensamiento por parte de los estudiantes, los cuales un $46,0 \%$ presentan conductas problemáticas moderadas; indicando que los estudiantes presentan una perspectiva poco común respecto a cómo piensa, la que está relacionado con problemas de personalidad (delirios o alucinaciones); por otro lado, un 31,8\% presentan conductas problemáticas bajas; mientras que, el restante $22,3 \%$ presentan conductas problemáticas severas. 


\section{Tabla 8}

Problemas emocionales y conductuales en la dimensión problemas de pensamiento

\begin{tabular}{lll}
\hline & $\mathbf{n}$ & $\mathbf{\%}$ \\
\hline Conductas problemáticas baja & 161 & 31,8 \\
Conductas problemáticas moderadas & 233 & 46,0 \\
Conductas problemáticas severa & 113 & 22,3 \\
Total & $\mathbf{5 0 7}$ & $\mathbf{1 0 0 , 0}$ \\
\hline
\end{tabular}

\section{Problemas emocionales y conductuales - Lesiones autoinfligidas}

En la tabla 9 se muestra los problemas emocionales y conductuales en la dimensión lesiones autoinfligidas, evidenciando un $27,6 \%$ y $9,5 \%$ en conductas problemáticas moderada y severa respectivamente, mostrando comportamientos autodestructivos que se puede denominar como: gesto suicida, intento o tentativa de suicidio o suicidio consumado; mientras tanto en conductas problemáticas baja obtuvieron un $62,9 \%$.

\section{Tabla 9}

Problemas emocionales y conductuales en la dimensión lesiones autoinfligidas

\begin{tabular}{lll}
\hline & $\mathrm{n}$ & $\%$ \\
\hline Conductas problemáticas baja & 319 & 62,9 \\
Conductas problemáticas moderadas & 140 & 27,6 \\
Conductas problemáticas severa & 48 & 9,5 \\
Total & 507 & 100,0 \\
\hline
\end{tabular}

\section{Prueba de normalidad}

En la presente tabla 10, con el propósito de realizar los análisis comparativos y contrastar las hipótesis planteadas, se procedió a realizar primero la prueba de bondad de ajuste para precisar si las variables presentan una distribución normal. En ese sentido, la tabla 21 presenta los resultados de la prueba de ajuste de Kolmogorov-Smimov (K-S), como se observa los datos correspondientes a las variables no presentan una distribución normal dado que el coeficiente obtenido (K-S) es significativo ( $\mathrm{p}<0.05)$. Por tanto, para los análisis estadísticos correspondientes se empleará estadística no paramétrica. 


\section{Tabla 10}

Prueba de normalidad sobre el uso problemático de Internet y problemas emocionales y conductuales

\begin{tabular}{|c|c|c|c|c|c|c|c|c|}
\hline \multirow{6}{*}{$\begin{array}{l}\text { Uso } \\
\text { problemátic } \\
\text { o de internet }\end{array}$} & Variables & Media & D.E & & K-S & & $\mathrm{P}$ & \\
\hline & Total & 7 & 21,2 & 8,470 & 8 & ,04 & 8 &, 00 \\
\hline & Depresión & & 14,0 & 4,705 & & ,11 & & ,00 \\
\hline & Rompimient & 4 & & 3,784 & 2 & & 0 & \\
\hline & $\begin{array}{ll}\mathrm{o} & \mathrm{de} \\
\text { reglas }\end{array}$ & 2 & 13,1 & $\begin{array}{l}4,343 \\
1,855\end{array}$ & 6 & ,08 & 0 & ,00 \\
\hline & $\begin{array}{l}\text { Conducta } \\
\text { agresiva }\end{array}$ & 1 & 13,2 & 2,852 & 5 & ,11 & 0 & ,00 \\
\hline $\begin{array}{l}\text { Problemas } \\
\text { emocionales }\end{array}$ & $\begin{array}{l}\text { Consumo de } \\
\text { alcohol y }\end{array}$ & & $\begin{array}{l}5,43 \\
8,42\end{array}$ & & 7 &, 25 & 0 & ,00 \\
\hline $\begin{array}{l}\text { y } \\
\text { conductuale } \\
\text { s }\end{array}$ & $\begin{array}{l}\text { tabaco } \\
\text { Problemas } \\
\text { somáticos }\end{array}$ & & & & 8 & ,12 & 0 & ,00 \\
\hline & $\begin{array}{l}\text { Problemas } \\
\text { de } \\
\text { pensamiento }\end{array}$ & & 5,15 & 2,196 & 4 & ,17 & 0 & ,00 \\
\hline & $\begin{array}{l}\text { Lesiones } \\
\text { autoinfligidas }\end{array}$ & & 3,94 & 1,650 & 5 & ,34 & 0 & ,00 \\
\hline & Total & & 63,3 & 15,71 & & ,08 & &, 00 \\
\hline
\end{tabular}

\section{Asociación entre las variables}

En la tabla 11 se aprecia una relación positiva media entre el uso problemático de internet con problemas emocionales y conductuales (rho=.185; $\mathrm{p}<.01$ ), es decir, los estudiantes que presentan un uso no problemático de internet a su vez presentan menores problemas emocionales y conductuales; respecto a la dimensión depresión, se observa que presenta una relación positiva media con el uso problemático de internet ( $\mathrm{rho=} .127 ; \mathrm{p}<.01)$, es decir, los estudiantes que presentan mayor depresión a su vez presentan un mayor uso problemático de internet. 


\section{Tabla 11}

Coeficiente de correlación entre las dimensiones de uso problemático de internet y problemas emocionales y conductuales

\begin{tabular}{lcc}
\hline & \multicolumn{2}{c}{ Uso problemático de internet } \\
\cline { 2 - 3 } & Rho & $\mathrm{P}$ \\
\hline Problemas emocionales y conductuales &, $185^{* *}$ &, 000 \\
Depresión &, $127^{* *}$ &, 004 \\
Rompimiento de reglas &, $226^{* *}$ &, 000 \\
Conducta agresiva &, $253^{* *}$ &, 000 \\
Consumo de alcohol y tabaco &, $210^{* *}$ &, 000 \\
Problemas somáticos &, $111^{*}$ &, 012 \\
Problemas de pensamiento &, $167^{* *}$ &, 000 \\
Lesiones autoinfligidas &, 048 &, 281 \\
& & \\
\hline
\end{tabular}

\section{Discusión}

La presente investigación ha explorado las variables uso problemático a internet y los problemas emocionales y conductuales; la revisión de la literatura permite comprender que el internet se ha convertido en una poderosa herramienta en la cual a medida que pasa el tiempo se reduce su uso en edades más tempranas, los niños y niñas con edades inferiores a 15 años penetran en este mundo por distintas razones (El Comercio, 2016). Asimismo, se entiende por problemas de conductas a aquellos patrones que se manifiestan a través de conductas agresivas o perturbadoras que pueden traer como consecuencia daños a otras personas como a sí mismas (Macià, 2007). En ese sentido, la presente investigación plantea que existe relación entre el uso problemático a internet y los problemas emocionales y conductuales en adolescentes, es así, que se pretende dar respuesta a los objetivos plateados mediante los resultados obtenidos.

En cuanto a la hipótesis general de la presente investigación, se comprobó la existencia de una relación significativa $(\mathrm{rho}=185 ; \mathrm{p}<.01)$ entre uso problemático de internet y problemas emocionales y conductuales, es decir, a mayor uso problemático de internet, mayores serán los problemas emocionales y conductuales. Estando en concordancia con lo expuesto por García (2008), donde menciona que los adolescentes expuestos al uso o abuso del internet pueden presentar cambios tanto en su conducta como en sus hábitos; Griffiths (1998) coincide con esta opinión afirmando que el uso desmedido de internet produce cambios en el estado de ánimo cuando 
empieza la actividad, como también la presencia de inquietud e irritabilidad cuando ya no se encuentra conectado o simplemente es interrumpido durante la conexión, en ocasiones ocurre todos estos síntomas cuando el individuo se encuentra en el proceso de controlar la frecuencia del uso de internet, no obstante, también se considera que el uso problemático de internet está relacionado con trastornos del control de impulsos o con los comportamientos compulsivos y obsesivos, ya que los síntomas del uso problemático de internet se relaciona con los criterios diagnósticos de estos trastornos.

Por su parte, Oliva (2007) considera que los adolescentes son más propensos a padecer de un tipo de adicción como en este caso al uso desmedido de internet debido a que su corteza prefrontal aún se encuentra inmaduro y esto equivale a que su autocontrol todavía no alcanzó al nivel de un adulto; Marks (1990), Potenza (2006) y Treuer, Fabián y Füredi (2001) refieren aquello que caracteriza a una adicción es la pérdida de control y la dependencia, teniendo en cuenta que en un inicio las actividades están controladas por reforzadores positivos, es decir el aspecto placentero, sin embargo, estas terminan descontrolándose por reforzadores negativos, especialmente el alivio de la tensión emocional, haciendo referencia de que una persona que controla el uso adecuado de internet, lo hace por la necesidad de comunicarse y especialmente por los beneficios que obtiene mediante su utilidad, mientras que una persona con un uso problemático realiza esta actividad de mantenerse conectado para aliviar su malestar emocional, ya sea aburrimiento, soledad, ira, nerviosismo, entre otros; por otro lado, Sánchez-Carbonell et al. (2008) y Cruzado, Muñoz y Navarro (2001) indican que la adición al internet trae consigo alteraciones en el bienestar psicológico presentado a través de múltiples problemas emocionales.

En cuanto a la primera hipótesis especifica se comprobó la existencia de una relación significativa (rho=.127; p<.004) entre uso problemático de internet y depresión, es decir, a mayor uso problemático de internet, mayor será la depresión. Estando en concordancia por lo expuesto por Echeburúa (2016) señala que el uso problemático del internet está íntimamente ligado con problemas de índole depresivo, discutiendo sobre la posibilidad de que este uso problemático sea fruto de problemas tanto depresivos como de otras índoles, tal como lo señala Betancourt y Andrade (2011) quienes sostienen que un adolescente con baja supervisión a nivel familiar es más vulnerable a problemas de índole depresivos ya que se agudizan en ellos sentimientos de soledad y abandono. Asimismo, Sánchez-Carbonell et al. (2008) recalcan que el uso problemático de internet modifica el estado de ánimo y genera depresión. 
En cuanto a la segunda hipótesis especifica se comprobó la existencia de una relación significativa (rho=.226; $\mathrm{p}<.000)$ entre el uso problemático de internet y rompimiento de reglas; es decir, a mayor uso problemático de internet, mayor será el rompimiento de reglas. Compartiendo la información expuesta por, Young (1996), Luengo (2004) y Sánchez-Carbonell et al. (2008) afirman que entre los criterios necesarios para un diagnóstico de adicción a internet es que el individuo es capaz de mentir o postergar actividades a fin de estar el mayor tiempo conectado. Del mismo modo, Echeburúa y De Corral (2010) refieren que, existen diversos riesgos de gran importancia con respecto al uso inapropiado o desmedido de las Tecnologías de Información y Comunicación (TIC's), es decir, es un medio por el cual nuestros adolescentes encuentran contenidos inapropiados, acoso o pérdida de la intimidad, además que a través de las redes pueden acceder a contenidos pornográficos y a su vez violentos que pueden manifestarse mediante mensajes racistas, relacionados a la anorexia e incitando al suicidio o muchas veces a la infracción de delitos.

En cuanto a la tercera hipótesis especifica se comprobó la existencia de una relación significativa (rho=.253; $\mathrm{p}<.000)$ entre el uso problemático de internet con conductas agresivas; es decir, a mayor uso problemático de internet, mayor será las conductas agresivas. Estando en concordancia con lo expuesto por García (2008) que afirma que a través de las páginas web los adolescentes se exponen a un sinfín de escenas violentas que resultan atractivas para muchos de ellos. Asimismo, Matalinares et al. (2013) demostraron que existe una relación significativa entre agresividad y adición a internet siendo los varones más agresivos que las mujeres tanto física como verbal. Además, Young (1996) señala que entre los elementos para el diagnóstico de la adicción a internet radica en la irritación en ausencia de conexión.

En cuanto a la cuarta hipótesis especifica se comprobó la existencia de una relación significativa $(r h o=.210 ; \mathrm{p}<.000)$ entre el uso problemático de internet y consumo de alcohol y tabaco; es decir, a mayor uso problemático de internet, mayor será el consumo de alcohol y tabaco. Estando en concordancia con lo expuesto por Ferreira y Torgal (2010) quien afirma que es el tabaco la primera droga que es consumida por los adolescentes, asimismo Díaz y Moral (2018) manifiestan que el consumo de alcohol ya forma parte de la cultura juvenil a pesar de ser ilegal su consumo en muchos lugares por parte de menores de edad.

Ko et al. (2008) y también Wartberg et al. (2016) muestran coincidencias en los resultados obtenidos mediante sus estudios que, los individuos que hacen un uso problemático de Internet 
tienen mayor probabilidad de presentar un consumo de riesgo de alcohol; en base a las afirmaciones mencionadas, Jessor (1991) mediante su Teoría de la Conducta Problema, refiere que existen distintos tipos de conductas inapropiadas, es decir, existiría una "propensión psicosocial" lo que conlleva a señalar que distintas conductas desviadas tiene relación con los rasgos de personalidad, contexto social, el factor ambiente percibido y por la misma conducta expresada del individuo; Ko et al. (2008) afirma que determinadas características psicosociales, como por ejemplo: ser del sexo masculino, disfunción familiar, baja autoestima y deficiente satisfacción de la vida, se encuentran asociados al consumo de alcohol como también a la adicción de Internet.

En cuanto a la quinta hipótesis específica se comprobó la existencia de una relación significativa (rho=.111; $\mathrm{p}<.012$ ) entre el uso problemático de internet y problemas somáticos; es decir, a mayor uso problemático de internet, mayor serán los problemas somáticos. Estando en concordancia con lo expuesto por Echeburúa (1999), Sánchez-Carbonell et al. (2008) y Luengo (2004) quienes afirman que, para el diagnóstico de la adicción a internet, es necesario evaluar la presencia de elementos que indiquen un desorden en la vida de las personas las cuales incluyen problemas de índole somáticos; es así que Didia et al. (2009) señala que el uso problemático de internet está asociado a dolores de espalda, migrañas, perturbación del sueño, entre otros.

En cuanto a la sexta hipótesis especifica se comprobó la existencia de una relación significativa (rho=.167; $\mathrm{p}<.000)$ entre el uso problemático de internet y problemas de pensamientos; es decir, a mayor uso problemático de internet, mayor serán los problemas de pensamientos. De acuerdo lo propuesto por Achenbach, Edelbrock y Howell los problemas de pensamiento están dentro del grupo de problemas denominado "internalizantes", el que está asociado con manifestaciones ansiosas (Alarcón \& Bárrig, 2015); por su parte, Lemos et al.(1992) encontraron que los problemas asociados con las patologías de tipo internalizantes se asemejan con los comportamientos propios del neuroticismo; según Eysenck (1990) los individuos con alto neuroticismo se caracterizan por ser ansiosos, depresivos, con baja autoestima y sentimientos de culpa (Schmidt, 2010).

Asimismo, durante el periodo de la adolescencia, se caracteriza por emociones negativas tales como la tristeza, vergüenza, ira y culpa, llevando a la persona a experimentar mayor susceptibilidad y malestar psicológico, no logrando controlar sus impulsos y evidenciando carencia de estrategias de afrontamiento y pensamiento irracionales (Ehrler, Evans \& McGhee, 1999). Considerando lo anterior mencionado, Estévez et al. (2009) señalaron que la impulsividad, 
el estado de ánimo vivenciado como desagradable, acompañado de inestabilidad del humor; la intolerancia los estímulos displacenteros, la timidez, baja autoestima, el estilo de afrontamiento inapropiado ante situaciones tensas; son características emocionales que incrementan la vulnerabilidad ante la adicción. Por otro lado, Sánchez-Carbonell et al. (2008) y Luengo (2004) estableciendo que las personas que se encuentran bajo la adicción al internet suelen desarrollar problemas del pensamiento ya que todos los pensamientos del individuo se verán vinculados al mundo digital convirtiéndose en el foco de atención de las personas.

En cuanto a la séptima hipótesis especifica los resultados señalan que no existe relación significativa entre $(\mathrm{rho}=.048 ; \mathrm{p}>.281)$ entre el uso problemático de internet y lesiones autoinfligidas; es decir, mayores niveles de uso problemático de internet no se relacionan con mayores o menores niveles de lesiones autoinfligidas. Dentro los problemas emocionales se encuentran las lesiones autoinfligidas, el cual se divide en dos categorías, así refiere Del Brío, Vásquez e Imaz (2019), estos son: intrapersonales o autoconcentradas, para la regulación de emociones y el autocastigo, en cuanto a las interpersonales, principalmente dirigidas a influenciar; asimismo se afirma que las personas que presentan estas conductas, suelen hacer uso de internet, y por otro lado, también tienden a cometer suicidio y las autolesiones; en cuanto a las lesiones autoinfligidas, estas se encuentran asociadas a diversos factores como inestabilidad emocional, síntomas depresivos y ansiosos, consumo de sustancias, trastornos de conducta alimentaria, trastorno de déficit de atención e hiperactividad, violencia familiar, abuso sexual, entre otras conductas de riesgo; se afirma que, cuando el adolescente se encuentra en un momento de vulnerabilidad tiende a iniciar con las autolesiones y estas se relacionan con factores internos (baja autoestima por verse diferente a los demás) mientras en cuanto a factor externo (exclusión social, bajo nivel económico y el uso inadecuado e irresponsable de las redes sociales).

\section{Conclusión}

En relación con los objetivos planteados, se encontró una correlación directa y positiva media entre el uso problemático de internet y problemas emocionales y conductuales (rho=.185; p=0,000) en estudiantes del nivel secundaria de una institución educativa pública de Lima Norte. Entendiendo que a mayor uso problemático de internet se presenta más problemas emocionales y conductuales. En base a lo presentado y la experiencia investigativa, se recomienda replicar la investigación con mayor cantidad de población para poder hacer generalizaciones considerando 
aspectos sociodemográficos diferentes a lo presentado. Se recomienda el estudio más minucioso y específico de cada dimensión de los problemas emocionales y conductuales en adolescentes como: depresión, rompimiento de reglas, conducta agresiva, consumo de alcohol y tabaco, problemas somáticos, problemas de pensamiento y lesiones autoinfligidas.

Asimismo, realizar estudios sobre el uso problemático de Internet relacionado a otros posibles problemas que pueda estar afectando el desarrollo integral de nuestros adolescentes, como: deserción escolar, pandillaje, conductas disruptivas, entre otros. También se recomienda estudiar el rol que ejerce las instituciones educativas con respecto a la prevención e intervención de problemas relacionados al desarrollo biopsicosocial de los adolescentes. Concientizar a los padres sobre la importancia del cuidado que deben tener con sus hijos respecto a estos problemas que afectan la salud mental de sus menores hijos. Crear programas de intervención, dirigido al grupo de estudiantes que participaron en la investigación, que brinde herramientas para erradicar o disminuir el uso problemático de internet.

\section{Referencias}

Alarcón, D. y Bárrig, P. (2015). Conductas internalizantes y externalizantes en adolescentes. Liberabit, 21 (2), 253-259. http://www.scielo.org.pe/pdf/liber/v21n2/a08v21n2.pdf

Araujo, E. D. (2015). De la adicción a la adicción a internet. Rev Psicolo Hered, 10 (2), 15. https://pdfs.semanticscholar.org/474b/5018fb5f9eaa1cc0d5e7a06cce2e5b640b0 6.pdf

Armas, M. (2010). Prevención e intervención ante problemas de conducta: estrategias para centros educativos y familias. Madrid: Wolters Kluwer Educación

Barreto, Y. (2018). Propiedades psicométricas de la Escala de Problemas Emocionales y Conductuales en Adolescentes de San Juan de Lurigancho, Lima 2018 [Tesis de licenciatura, Universidad César Vallejo]. http://repositorio.ucv.edu.pe/bitstream/handle/UCV/31959/Barreto_MY.pdf?sequ ence $=1 \&$ isAllowed $=\mathrm{y}$

Bermúdez, P. (2018). Súper interesantes cifras de la penetración de internet y facebook en el Perú y el mundo. https://gestion.pe/blog/revoluciondigital/2018/03/super-interesantes-cifras-delapenetracion-de-internet-yfacebook-en-el-peru-y-el-mundo.html?ref=gesr 
Betancourt, D. y Andrade, P. (2011). Control parental y problemas emocionales y de conducta en $\begin{array}{lllll}\text { adolescentes. Revista Colombiana de Psicología, } 20 & \end{array}$ https://www.redalyc.org/articulo.oa?id=804/80419035006

Chang, J. P. C., y Hung, C. C. (2017). Uso problemático de internet. http://iacapap.org/wp-content/uploads/H.6-Uso-problem\%C3\%A1tico-deInternetSPANISH-2017.pdf

Cheung, L., y Wong, W. (2011). The effects of insomnia and internet addiction on depression in Hong Kong Chinese adolescents: an exploratory cross-sectional analysis. Sleep and Internet Addiction, 20, 311-317. https://doi.org/10.1111/j.1365-2869.2010.00883.x

Comisión Económica para América Latina y el Caribe (CEPAL) (2015). La nueva revolución digital: de la Internet del consumo a la Internet de la producción. https://repositorio.cepal.org/bitstream/handle/11362/38604/4/S1600780_es.pdf

Cruz, I. (2015). El uso y los significados de las NTICS en la sociedad informatizada. https://books.google.com/books/about/El_uso_y_los_significados_de_las_N

Cruzado, J., Muñoz, M. y Navarro, E. (2001). Adicción a Internet: de la hipotética entidad diagnóstica a la realidad clínica. Psicología clínica, legal y forense, 1 (2), 93-102. https:/doi.org/10.1074/jbc.M109.041368

Custodio, M. (2018). Problemas emocionales y de conducta: Estudio realizado con los estudiantes de quinto grado de primaria de la Escuela José Joaquín Palma de la ciudad de Quetzaltenango [Tesis de licenciatura, Universidad Rafael Landívar]. http://recursosbiblio.url.edu.gt/tesisjrcd/2018/05/22/Custodio-Maria.pdf

Del Brío, P., Vásquez, M. \& Imaz, C. (2019). Adolescente con autolesiones no suicidas en un entorno de adversidad psicosocial. Arch. Argent. Pediatr., 117 (5), 485-488. http://dx.doi.org/10.5546/aap.2019.e485

Díaz, N. y Moral, M.V. (2018). Consumo de alcohol y conducta antisocial e impulsividad en adolescentes españoles. Acta colombiana de Psicología, 21 (2), 121-130. http://www.scielo.org.co/pdf/acp/v21n2/en_0123-9155-acp-2102-110.pdf

Didia, J., Dorphinghaus, A., Maggi, C. \& Haro, G. (2009). Adicciones a Internet: Una posible inclusión en la nosografía. Revista de Psiquiatría del Uruguay, 73, 73-82. http://www.spu.org.uy/revista/ago2009/02_TO_06.pdf 
Echeburúa, E. (1999). ¿Adicciones sin drogas? Las nuevas adicciones. Bilbao: Desclée de Brouwer.

https://www.academia.edu/31792963/A_Adicciones_Sin_Drogas_Las_Nuevas_A dicciones_Juego_Sexo_Co

Echeburúa, E. \& De Corral, P. (2010). Adicción a las nuevas tecnologías y a las redes sociales en jóvenes: un nuevo reto. Adicciones, 22 (2), 91-96. http://www.adicciones.es/index.php/adicciones/article/view/196

Echeburúa, O. E. (Ed.). (2016). Abuso de internet: ¿antesala para la adicción al juego de azar online? España: Ediciones Piramide.

http://www.biblioteca.cij.gob.mx/Archivos/Materiales_de_consulta/Drogas_de_Ab uso/Articulos/Abuso\%20de\%20internet.pdf

Ehrler, D. J., Evans, J. G., \& McGhee, R. L. (1999). Extending big-five theory into childhood: A preliminary investigation into the relationship between big-five personality traits and behavior problems in children. Psychology in the Schools, 36, 451-458. 10.1002/(SICI)15206807(199911)36:6<451: AID-PITS1>3.0.CO;2-E

El Comercio, Diario. (2016). Unicef: el 42\% de menores de 15 años se conecta a Internet en el país. https://elcomercio.pe/peru/unicef-familias-deben-generarentorno-virtual segurojovenes-noticia-605140-noticia/

Escurra L. (1998) Cuantificación de la validez de contenido por criterio de jueces. Revista de Psicología, 6 (1), 103-110. http://revistas.pucp.edu.pe/index.php/psicologia/article/view/4555/4534

E. Echeburúa, F. J. Labrador \& E. Becoña (Eds.) (2009). Adicción a las nuevas tecnologías en adolescentes y jóvenes. Madrid: Pirámide

Ferreira, M. \& Torgal, M. (2010). Consumo de Tabaco y alcohol en la adolescencia. Revista Latino-Americana de Enfermería, 18 (2). http://www.scielo.br/pdf/rlae/v18n2/es_17.pdf

Garaigordobil, M. \& Maganto, C. (2013). Problemas emocionales y de conducta en la infancia: Un instrumento de identificación y prevención temprana. Padres y Maestros/Journal of Parents and Teachers, 1 (351), 34-40. https://revistas.comillas.edu/index.php/padresymaestros/article/view/1050/894

García, A. (2008). Riesgos del uso de internet por niños y adolescentes. Estrategias de seguridad. Acta Pediátrica de México, 29 (5), 272-278. 
http://www.redalyc.org/pdf/4236/423640313006.pdf

García, C., López, M. \& García, A. (2014). Los riesgos de los adolescentes en Internet: los menores como actores y víctimas de los peligros de Internet. Revista Latina de Comunicación Social, 69, 462-485. Doi: 10.4185/RLCS-2014-1020

Gonzáles, G. (2012). Protocolo para tratar la adicción a internet. https://fundacionesplai.org/tucontrolas/2012/03/26/protocolo-para-tratar-laadiccion-ainternet/

Gackenbach, J. (Ed.) (1998). Psychology and the internet: Interpersonal, interpersonal and transpersonal applications. New York: Academic Press.

Griffiths, M. (1999). A 'components' model of addiction within a biopsychosocial framework. Journal of Substance Use, 10, 191-197. http://www.academia.edu/429550/

Griffiths M. D. 2005. A components model of addiction within a biopsychosocial framework. Journal of Substance Use, 10 (4): 191-197. https://www.addictionpsychologist.org/wpcontent/uploads/2019/03/griffiths-2005-addiction-components.pdf

Hernández, L., Del Palacio, A., Freyre, M., \& Alcázar, R. (2011). La perspectiva dimensional de la psicopatología. Revista mexicana de Psicología, 28 (2), 110-120. http://www.redalyc.org/articulo.oa?id=243029631001

Hernández, R., Fernández, C. \& Baptista, P. (2014). Metodología de la investigación. México: McGraw-Hill

Herrera, P. (1999). Principales factores de riesgo psicológicos y sociales en el adolescente. Revista $\begin{array}{lllll}\text { Cubana } & \text { de } & \text { Pediatría, } & \text { 39-42. }\end{array}$ http://scielo.sld.cu/scielo.php?script=sci_arttext\&pid=S003475311999000100006 \&lng=es\&tlng=es

Instituto Nacional de Estadística e Informática (INEI). (2018). Estadísticas de las Tecnologías de Información y Comunicación en los Hogares en el trimestre de enero-febrero-marzo 2018 (Informe Técnico No 2). Lima: INEI.

https://www.inei.gob.pe/media/MenuRecursivo/boletines/01-informe-tecnico n02_tecnologias-de-informacion-ene-feb-mar2018.pdf 
Jessor, R. (1991). Risk behavior in adolescence: A psychosocial framework for understanding and action. Journal of Adolescent Health, 12 (8), 597-605. https://doi.org/10.1016/1054139X(91)90007-K

Ko, C. H., Yen, J. Y., Yen, C. F., Chen, C. S., Weng, C. C. \& Chen, C. C. (2008). The association between Internet addiction and problematic alcohol use in adolescents: The problem behavior model. CyberPsychology \& Behavior, 11 (5), 571-576. Doi: 10.1089/ cpb.2008.0199

Lemos, S., Fidalgo, A., Clavo, P. \& Meléndez, P. (1992). Salud Mental de los adolescentes asturianos. Psicothema, 4 (1), 21-48. http://www.psicothema.com/psicothema.asp?id=813

Luengo, A. (2004). Adicción a Internet: conceptualización y propuesta de intervención.

Revista Profesional Española de Terapia Cognitivo Conductual, 2, 22-52. http://www.jogoremoto.pt/docs/extra/BL5L6u.pdf

Macià, D. (2007). Problemas cotidianos de conducta en la infancia: Intervención psicológica en el ámbito clínico y familiar. Madrid: Pirámide.

Marks, I. (1990). Behavioural (non-chemical) addictions. British Journal of Addiction, 85 (11), 1389-1394. https://doi.org/10.1111/j.13600443.1990.tb01618.x

Matalinares, M., Arenas, C., Díaz, G. \& Dioses, A. (2013). Adicción a la internet y agresividad en estudiantes de secundaria del Perú. Revista de Investigación en Psicología, 16 (1), 75-93. https://revistasinvestigacion.unmsm.edu.pe/index.php/psico/article/download/392 $0 / 3144 / 0$

Mellizo, P. (2017). Uso problemático del Internet y autoestima en adolescentes: una revisión de la literatura [Tesis de licenciatura, Universidad del Rosario]. http://repository.urosario.edu.co/bitstream/handle/10336/14165/Uso\%20Problem \%C3\%A1tico\%20del\%20Internet\%20y\%20Autoestima\%20en\%20adolescentes.p df? sequence $=1 \&$ is Allowed $=\mathrm{y}$

Moral, M. D. \& Fernández, S. (2019). Uso problemático de internet en adolescentes españoles y su relación con autoestima e impulsividad. Avances en psicología latinoamericana, 37 (1), 103-119.

https://dialnet.unirioja.es/descarga/articulo/6805347.pdf 
Oliva, A. (2007). Desarrollo cerebral y asunción de riesgos durante la adolescencia. Apuntes de Psicología, 25 (3), 239-254. https://psicopedia.org/wpcontent/uploads/2014/06/Riesgosen-la-adolescencia.pdf

Potenza, M. (2006). Should addictive disorders include non-substance related conditions? Addiction, 101 (1), 142-151. Doi: 10.1111/ j.13600443.2006. 01591.x

Rial, A., Golpe, S., Gómez, P. \& Barreiro, C. (2014). Variables asociadas al uso problemático de internet entre adolescentes. Salud $y$ drogas, $15 \quad$ (1), 25-38. https://www.redalyc.org/pdf/839/83938758003.pdf

Salavera, C. \& Usán, P. (2019). Influencia de los problemas internalizantes y externalizantes en la autoeficacia en estudiantes de Secundaria. Revista de investigación educativa, 37 (2), 413429. Doi: http://dx.doi.org/10.6018/rie.37.2.323351

Sánchez, L., Crespo, G., Aguilar, R., Bueno, F., Benavente, R., \& Valderrama, J. (2015). Los adolescentes y las tecnologías de la información y la comunicación. Guía de Padres ayudándoles a evitar riesgos. España: Plan Municipal de Drogodependencias, Unidad de Prevención Comunitaria de Conductas Adictivas. http://digital.csic.es/bitstream/10261/132633/1/TICPadres.pdf

Sánchez-Carbonell, X., Beranuy, M., Castellana, M., Chamarro, A. \& Oberst, U. (2008).

Adicción a Internet y móvil: ¿Moda o trastorno? Adicciones, 20 (2), 149-160. https://www.researchgate.net/publication/215508643_La_adiccion_a_Internet_y_ al_movil_Moda_o_trastorno

Schmidt, V. (2010). Las bases biológicas del neuroticismo y la extraversión ¿por qué nos comportamos como lo hacemos? Revista Latinoamericana de Ciencia Psicológica, 2 (1), 20-25. https://www.redalyc.org/pdf/3331/333127086005.pdf

Treuer, F., Fabian, Z. \& Furedi, J. (2001). Internet addiction associated with features of impulse control-disorder: is it a real psychiatric disorder? Journal of Affective Disorders, 66 (2-3), 283. Doi: 10.1016 / S0165-0327 (00) 00261-5

Valencia, M. R. \& Andrade, P. (2005). Validez del Youth Self Report para problemas de conducta en niños mexicanos. International Journal of Clinical and Health Psychology, 5 (3), 499520. https://www.redalyc.org/pdf/337/33705306.pdf

Wartberg, L., Brunner, R., Kriston, L., Durkee, T., Parzer, P., Fischer-Waldschmidt, G. \& Kaess, M. (2016). Psychopathological factors associated with problematic alcohol and problematic 
Internet use in a sample of adolescents in Germany. Psychiatry Research, 240, 272-277. Doi: 10.1016/j.psychres.2016.04.057

Young, K. S. (1996). Internet Addiction: The emergence of a New Clinical Disorder. CyberPsychology \& Behavior, 1 (3), 237-244. Doi: https://doi.org./10.1089/cpb.1996.1.237 